\title{
Stealth Strategies of Mycobacterium tuberculosis for Immune Evasion
}

\author{
Muhammad Ahsan Naeem ${ }^{1}$, Waqas Ahmad ${ }^{1}$, Rohit Tyagi $^{2}$, Qaiser Akram¹, \\ Muhammad Younus ${ }^{1}$, Xilin Liu ${ }^{3 *}$ \\ ${ }^{1}$ University College of Veterinary and Animal Sciences, Narowal, 51600, Pakistan \\ ${ }^{2}$ College of Veterinary Medicine, Huazhong Agricultural University, Wuhan \\ 430070, China \\ ${ }^{3}$ Department of Hand Surgery, China Japan Union Hospital, Jilin University, 126 \\ XianTai Street, Changchun, China \\ *Xilin Liu liuxilin@jlu.edu.cn
}

DOI: https://doi.org/10.21775/cimb.041.597

\begin{abstract}
Tuberculosis is a devastating disease causing high mortality all over the world, especially in the developing countries. Mycobacterium tuberculosis (M. tb) is the causative agent of tuberculosis which replicates in the intracellular environment of host macrophages. Although the host immune system is capable of completely eliminating the pathogen, co-evolution of $M$. $t b$ with humans has resulted in its ability to hijack the host innate and adaptive immune systems in numerous ways. Limited recent progress has been made in the understanding of $M$. tb immune escape mechanisms, hence exploration of survival strategies of $M$. tb has been critically reviewed with an insight into understanding its pathogenesis. We summarized the recent studies regarding the modulation of innate immune response, adaptive immune response, epigenetics and the role of miRNA. All of these advancements suggest that $M$. tb is well-familiarize with the host immune system and possess the ability to hijack it for intracellular survival.

\section{Introduction}

Tuberculosis (TB) is described in almost all of the recorded history of humanity (Comas et al., 2013). It is the leading cause of death due to infectious disease and, therefore, has been enlisted in the priority list of OIE and WHO (Dean et al., 2018; Lisdawati et al., 2015). Multidrug-resistant (MDR) and extensively drugresistant (XDR) TB have contributed greatly in treatment failure and increasing case fatalities (Dean et al., 2018; Lisdawati et al., 2015). M. tb has been evolved to endure many different environments ranging from soil dweller to intracellular pathogen (Cambier et al., 2014). While infecting macrophages and dendritic cells (DCs), M. tb hijacks them for its survival and dissemination (Harding and Boom,
\end{abstract}


2010). It further inhibits the major histocompatibity complex class-II (MHC-II)mediated antigen presentation to adaptive immune cells, i.e. $\mathrm{CD}^{+} \mathrm{T}$ cells (Harding and Boom, 2010). To survive in the hostile cellular environment, pathogenic mycobacteria employ various strategies, such as: phago-lysosome maturation inhibition, autophagy suppression, cytokine activation and production, reactive oxygen (ROS) and nitrogen species (RNS) inhibition, manipulation of antigen presentation to T cells, and epigenetics (Cambier et al., 2014; Harding and Boom, 2010). M. th is recognized by macrophages through pattern recognition receptors (PRRs) which include; Toll-like receptors (TLR-2, TLR-4, TLR-9), NOD-like receptors (NLR), and C-type lectin receptor (CLR) (Mortaz et al., 2015). Recognition activates intracellular signaling cascades, such as IL-1 receptor-associated kinases (IRAK), TNF receptor-associated factor 6 (TRAF-6), TGF-ß-activated protein kinase 1 (TAK1), and mitogen-activated protein kinases (MAPK) (Ernst, 1998; Mortaz et al., 2015). TLR signaling leads to the release of pro-inflammatory cytokines, such as tumor necrosis factor (TNF), interleukin-12 (IL-12), and IL-1 $\beta$. These cytokines recruit adaptive immune cells, T-cells, which can aid in the mycobactericidal response by their interferon-gamma (IFN- $\gamma$ ) release (Mortaz et al., 2015). IFN- $\gamma$ increases the microbicidal capacity of macrophages by enhancing trafficking of the phagocytosed bacteria to lysosomes. Moreover, IFN- $y$ triggers macrophages to produce damaging radical species, such as ROS and RNS (Mortaz et al., 2015). How M. tb successfully manipulate these responses of the innate and adaptive immune system of host for its persistence is questionable till date? Similar to our present study on $M$. $t b$, Ahmed et al., (Ahmed et al., 2016) has described the similar stealing strategies of another notorious intracellular organism, Brucella. The present review will discuss the current knowledge of $M$. tb survival mechanisms to withstand host cell defenses.

\section{Modulation of innate immunity}

Innate immunity is necessary to understand the pathogenicity of intracellular bacteria, such as: M. tb, Salmonella spp. etc. Hurley et al., (Hurley et al., 2014) has described the interaction of Salmonella enterica with human host regarding activation of innate immune system. They also suggested that this interaction is necessary for the survival of $S$. enterica within innate immune cell i.e. macrophage. In case of $M$. tb, the role of innate immunity initiates when an individual inhales tubercle bacilli. Macrophages make up the first line of defense against M. tb (Figure 1) (Ferraris et al., 2018; Hossain and Norazmi, 2013). They eliminate the phagocytosed bacteria by fusing phagosome with lysosomes, which contain acidic and hydrolyzing enzymes (Ferraris et al., 2018). Although complement pathways are activated during $M$. tb infection yet the bacteria counter or manipulate the host immune system in a very shrewd way. Alternate complement pathway is activated when the $M$. tb infection happened from noninhalation route, whereas, classical pathway activated when the infection occur from inhalation (lung) route (Ferguson et al., 2004). Normally, innate immune cells, macrophages and dendritic cells (DCs), engulf bacteria and present their epitopes to helper (HTL) and cytotoxic T-lymphocytes (CTL) (Ferraris et al., 2018). This process triggers different signaling pathways in the host cell. These 
epitopes drive HTL/CTL priming and memory generation and induce host adaptive immune response.

Recent studies have suggested that phagosomal maturation inhibition is important for the progression of infection, which would otherwise lead to bacterial clearance and antigen processing (Torres et al., 2006). Thus, several proteins in $M$. tb play a role in the phagosomal maturation inhibition. For example, Rv0198c (Zmp1), a culture filtrate (secretory) protein that functionally acts as a $\mathrm{Zn}$ metalloproteinase, inhibits the conversion of pro-IL-1 $\beta$ to IL-1 $\beta$ by inhibiting caspase-1. Consequently, this suppresses IL-1 $\beta$-mediated inflammasome activation and phagosomal maturation (Figure 1) (Master et al., 2008). Phagolysosomal fusion is also inhibited by exotoxins/proteasomes, such as SapM (Rv3310), acid phosphatase/ phosphomonoesterase, and serine/ threonine kinase (PknG- Rv0410c) (Walburger et al., 2004). Furthermore, a cell wall component, lipoarabinomannan (LAM), has been reported to inhibit phagolysosomal fusion. This inhibition occurs through the release of phosphoinositol-3-phosphate phosphatase enzyme from $M$. th that acts against host PI3P, which is recruited to phagosomal membranes to attract lysosomes for fusion (Deretic et al., 2006). Similarly, another cell wall component, trehalose dimycolate (TDM), has also been implicated in delaying phagosome maturation. TDM is recognized by CLR (Lang, 2013), TLR2, CD14, and the scavenger receptor, MARCO (Bowdish et al., 2009). TDM modulates mincle signaling, which triggers pro-inflammatory (TNF- $\alpha$ and NO) and anti-inflammatory (IL-10) cytokines by syk/CARD9 signaling in mouse bone marrow-derived macrophages (mBMDM) (Patin et al., 2017). Recently, Patin et al. (Patin et al., 2017) have experimentally shown the delaying of phagosomal maturation by TDM. Using an IgG antibodyopsonised bead model, they studied FcrR-mediated phagosomal formation. TDM triggered an inhibitory cross-talk between mincle and the FcrR, with the end result of delayed phagosomal maturation (Patin et al., 2017). Other virulence factors negatively modulate pathogen recognition receptors (PRRs), and more specifically, TLR2, signaling. These include; 60 kDa chaperonin-2 (GroEL2), sulfated glycolipids, $6 \mathrm{kDa}$ early secretory antigen target (ESAT-6), and lipoprotein LpqT (Figure 1). Signals from TLR2 activate the transcriptional activator, NF-kB, for innate immune cytokine release, phagosome maturation, and apoptosis, which are all inhibited by these virulence factors (Li et al., 2018; Naffin-Olivos et al., 2014).

Another strategy for $M$. th is to manipulate innate immunity indirectly. An example is that $M$. tb recruits the host protein, TACO1/CORO1A, to its phagosome, which also prevents phago-lysosomal fusion (Figure 1) (Jayachandran et al., 2007; Meena, 2010; Pieters, 2008). In non-infected macrophages, Coronin 1 is distributed equally between the cytosol and membranes. It is exclusively recruited to the phagosomal membrane surrounding live but not dead $M$. th in macrophages. In macrophages containing dead $M$. tb, coronin 1 is initially cointernalized but rapidly dissociates from phagosomes and non-coated phagosomes fuse with lysosomes, resulting in the degradation of mycobacteria. Hence, it suggests an essential role for coronin-1 in preventing the fusion of phagosomes with lysosomes (Ferrari et al., 1999; Tailleux et al., 2003). However, 
this recruitment of coronin-1 is specific to alveolar macrophages only and does not liable for Kupffer cells, the resident macrophages of the liver (Ferrari et al., 1999). Regarding molecular activities of coronin-1 in macrophages, these are fully dispensable on F-actin-mediated functions, which are analyzed by phagocytosis, micropinocytosis and motility. Coronin-1 is required for the activation of the $\mathrm{Ca}^{2+}$ dependent phosphatase and calcineurin (Jayachandran et al., 2007). In wild-type macrophages calcineurin becomes activated upon internalization of $M$. th and blocks phagosome-lysosome fusion by an unknown mechanism, allowing $M$. tb survival. In the absence of coronin-1, calcineurin activation does not happen inhibiting phagosome-lysosome fusion and intracellular degradation of the phagocytosed mycobacteria (Jayachandran et al., 2007). Similarly, activation of tyrosine phosphatase (PtpA), inhibit the recruitment of vacuolar proton-ATPase to the mycobacterium-containing phagosome membrane (Figure 1) (Wong et al., 2011). This prevents lysosomal acidification despite the membrane surface containing molecular markers (CD63, LAMP-1, LAMP-2, transferrin receptors, and Rab5) for fusion with lysosomal membranes (Chen et al., 2015; Gidon et al., 2017).

M. $t b$ survives and replicates within the acidic environment of lysosome by engulfment in macrophages via Fcr receptors (Levitte et al., 2016) and Rv3671c (MarP: membrane serine protease), an acid endurance and virulence providing factor (Levitte et al., 2016). Another host factor, which is an immunosuppressive, non-classical, and class Ib molecule, is Human Leukocyte Antigen-G (HLA-G). This factor leads to the induction of apoptosis, suppression of cytotoxicity, and modulation of cytokine secretion (Saurabh et al., 2018). This immunosuppressive function is highly conducive for $M$. tb evasion from host responses. Countering this strategy by blocking HLA-G leads to upregulation of pro-inflammatory cytokines (IFN- $\gamma$ and TNF- $\alpha$ ). This upregulation can be observed in $M$. th antigenspecific CD3 ${ }^{+}$T cells in TB-pulmonary effusion patients (Saurabh et al., 2018). Similarly, when host immunity dominates over the pathogen's virulence, innate immune cells present antigen to $\mathrm{CD}^{+}{ }^{+} \mathrm{T}$ cells by the MHC-II pathway. This culminates in the development of a Th1 response with release of IFN-Y (Rohde et al., 2007). Here, M. tb uses the host's own negative co-stimulatory receptor, i.e. lymphocyte activation gene-3 (LAG-3). LAG-3 favors $M$. th survival by interfering with the mitochondrial apoptosis pathway. Thus, the silencing/down-regulation of LAG-3 in CD4 ${ }^{+} \mathrm{T}$ cell leads to an increase in $M$. tb killing, IFN- $\gamma$ expression and decrease in mitochondrial electron transport (Phillips et al., 2017).

Intracellular $M$. tb can also survive in the acidic environment of the phagolysosome partially by intrinsic characteristics. For example, mycolic acid forms the thick and waxy cell wall that provides a physical barrier against the entry of protons of acidic media (Saviola, 2013). Some environmental mycobacteria ( $M$. smegmatis), can naturally grow in acidic condition (Saviola, 2013) while pathogenic mycobacteria $(M . t b)$ has also evolved acid tolerance (Saviola, 2013). Genes regulating cell wall structure also upregulate during contact with acidic environments, genes involved in cell wall structure, such as mymA operon, becomes up-regulated (Singh et al., 2005). On the other hand, the production and storage of triacylglycerol starts in an extended period of acidic 


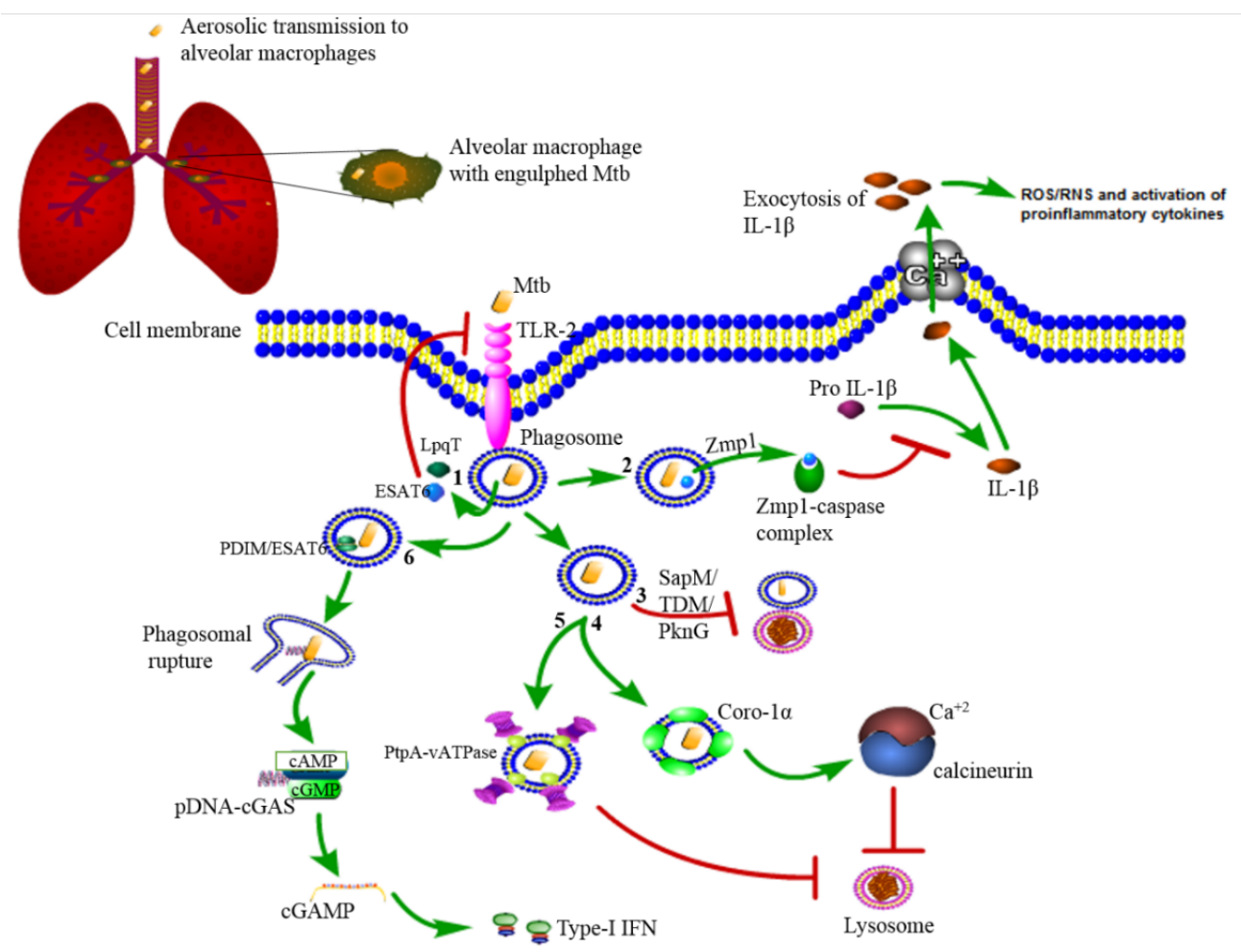

Figure 1. Schematic illustration of $M$. tb-host interactions with and inside of the alveolar macrophage. M. tb processing in alveolar macrophages depicted by its step-wise strategies for its intracellular lifestyle. 1) Membranous invagination to entrap M. tb within a phagosome. ESAT- 6 and LpqT are involved in the inhibition of TLR2 signaling-led recognition and response to invading $M$. tb. 2) Zmp1 is secreted by phagosomal $M$. tb into the cytosol to complex with/block activated caspase-1. This complex ultimately prevents oxidative stress responses by inhibiting the conversion of pro-IL-1 $\beta$ to IL-1 $\beta$. 3) Phagosomal M. tb releases protease (SapM), cell wall component (TDM), and kinase (PknG) to inhibit phagosome-lysosme fusion. 4) Phagosome-lysosome fusion is also inhibited by coating phagosomal membrane with host protein coro-1a. Coro-1a activates calciumcalcineurin complext and blocks the fusion of the coated phagosome with lysosomes. 5) Activation of bacterial factor, tyrosine phosphatase (PtpA), inhibits the recruitment of vacuolar proton-ATPase to the membrane of Mycobacterium-containing phagosomes. This ultimately blocks its fusion with lysosome. 6) Secretion of EsxA and PDIM led to pore formation in phagosomal membranes. $M$. tb immediately senses the cytosolic DNA sensor, cyclic GMP-AMP synthase (cGAS), which leads to the synthesis of the second messenger, cGAMP. The ultimate target of CGAMP is to release the pathogen growth-favoring cytokines, type I IFNs.

stress in M. tb (Purdy et al., 2013). Triacylglycerol also acts as an energy source to bacilli during the latent phase, representing an important strategy of survival (Purdy et al., 2013).

Another strategy for intracellular survival used by $M$. tb is through the interaction of tyrosine phosphatase, PtpA, with the host ubiquitin system. PtpA inhibits innate immunity by interacting with the host protein, TRIM27 (Wang et al., 2016). 
TRIM27 is reported to possess a conserved RING domain and usually acts as an E3 ubiquitin ligase that promotes apoptosis in macrophages. Thus, this TRIM27PtpA interaction could be a potential target for new therapeutic strategies against TB (Wang et al., 2016).

\section{Modulation of Adaptive Immunity}

During M. tb infection, optimal T cell activation is possible by transferring $M$. $t b$ from the lung to a local draining lymph node (Srivastava and Ernst, 2014). M. tbinfected monocyte-derived dendrictic cells are less efficient at activating CD4 ${ }^{+} \mathrm{T}$ cells because of bacterial inhibition of antigen presentation. Two types of $M$. tb antigens, such as soluble, non-degraded protein (Srivastava and Ernst, 2014) and lipid antigens (De Libero and Mori, 2014), are directly released from the infected innate immune cells. These antigens are presented to local lymph nodes for the priming of $\mathrm{T}$ lymphocytes (Figure 2). Lipid antigens are recognized by CD1 molecules and interact with T-cell receptors (TCRs) to activate T-cells (De Libero and Mori, 2014). There are different lipid antigens for the five types (a-e) of CD1 molecules. Some of the lipid antigens recognized by different CD1 molecules include: dideoxymycobactin (precursor of mycobactin and siderophore to scavenge iron), glycerol monomycolate, phosphatidylinositol mannoside (PIM), lipoarabinomannan (LAM), lipomannan, diacylated sulfoglycolipids, methyllysophosphatidic acids (mLPA), mannosyl $\beta$-1-phosphomycoketides (MPM) and diphosphatidylglycerol (DPG) (De Libero and Mori, 2014). The identification of protein or lipid antigens for $M$. tb virulence is essential, because it becomes the basis for vaccine development (De Libero and Mori, 2014). There is high hope that the involvement of these candidates in new vaccine trials would lead to an effective vaccination against TB (De Libero and Mori, 2014).

Studies from the Wang et al., (Wang et al., 2015) laboratory described the molecular mechanisms involved in the inhibition of T-cells during $M$. tb infection. Upon active infection, CD244 receptors were upregulated with associated signaling in $\mathrm{CD}^{+} \mathrm{T}$ cells (Wang et al., 2015). As a result, there is blockage in the production of IFN- $\gamma$ and TNF- $\alpha$. This is due to the production and release of high levels of a long, noncoding RNA (IncRNA)-BC050410 [called IncRNAASGSTT1(1-72) or IncRNA-CD244] in the CD244 ${ }^{+} C D 8^{+}$T-cell subpopulation (Wang et al., 2015). CD244 signaling at IncRNA-CD244 locus maintains a permissive chromatin state and hence leads to IncRNA-CD244 expression. On contrary, EZH2, polycomb protein enhancer of zeste homolog 2, positioning at IFN- $\gamma / T N F-\alpha$ promoters leads to a repressive chromatin state of IFN- $\gamma / T N F-\alpha$ loci. This occurs by IncRNA-CD244-mediated H3K27 trimethylation at the IFN-y/TNF- $\alpha$ loci (Wang et al., 2015). This inhibits IFN- $/$ /TNF- $\alpha$ expression in CD8 ${ }^{+} \mathrm{T}$ cells. This inhibition of IFN-y and TNF- $\alpha$ release can be reversed by knock down of IncRNA-CD244 or by blocking the CD244 receptor (Wang et al., 2015).

\section{M. tb stealthy behavior for virulence}

3.1 Strategy to break from the membranous prison: Access to the cytosol

$M$. tb invasion into the cytosolic compartment is highly decisive for both the host and the pathogen. PDIM, together with EsxA (ESAT-6), plays an active role in phagosomal membrane rupture in host cells (Augenstreich et al., 2017). The 


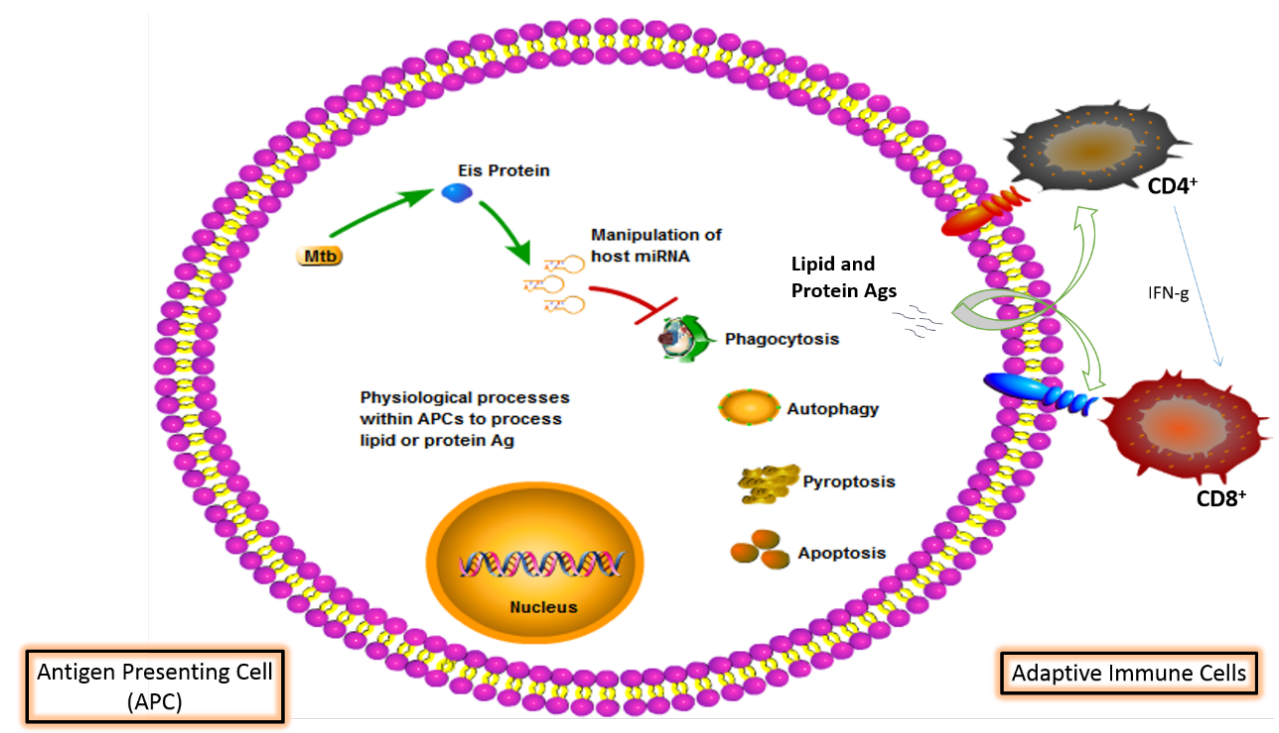

Figure 2. Epigenetic control over innate immune cell physiological mechanisms. The Eis protein of $M$. tb induces miRNA directly involved in regulation of cell physiological pathways, such as phagocytosis, autophagy, pyroptosis, and apoptosis. These physiological pathways are involved in antigen ( $\mathrm{Ag}$ ) processing. Inhibition of these pathways ultimately blocks or delays antigen Ag processing for T-lymphocytes.

study concluded that the cytosolic escape of $M$. tb is correlated with the production and export of phthiocerol dimycocerosates (DIM/PDIM) (Augenstreich et al., 2017). The secretion of Esx-1 and PDIM led to pore formation and phagosomal membrane disruption (Augenstreich et al., 2017). M. tb immediately senses the cytosolic DNA sensor, cyclic GMP-AMP synthase (cGAS), which leads to the synthesis of second messenger, cGAMP. The ultimate target of cGAMP is to release the bacterial pathogen-favoring cytokines, i.e. type I IFNs (as shown in Figure 1), via stimulator of IFN genes (STING) and the TBK-1-IRF-3-IFN- $\beta$ signaling cascade (Watson et al., 2015). The presence of DIM/PDIM also favors $M$. $t b$ replication by arresting phagosomal acidification (Augenstreich et al., 2017).

\subsection{Strategy to dampen oxidative damage}

The phagolysosome is basically a toxic environment for pathogens (Swanson, 2002), as it contains reactive oxygen intermediates (ROIs) (Ehrt and Schnappinger, 2009). To combat these intra-phagolysosomal stressors, interruption of the oxidative cascade is important for $M$. $t b$. For example, mycothiol, a functional analog to host glutathione, can become oxidized at its cysteine residues to form mycothione, preventing oxidation of other mycobacterial molecules (Saviola, 2010). Moreover, host cell glutathione is detrimental for $M$. $t b$, as it disturbs the oxidation-reduction balance of the pathogen, so it is neutralized by bacterial superoxide dismutase (SOD) and catalase (KatG) (Saviola, 2010). The toxic effects of oxidative radicals on the pathogen's DNA induces modification of its compactness and structural changes. These changes are countered by $M$. 
tb histone-like protein, LSR2, by acting as a physical barrier to protect the DNA (Colangeli et al., 2009).

\subsection{Divalent metal homeostasis}

\subsubsection{Esx-3 and divalent metal homeostasis}

ESX-3 is essential for optimal growth of $M$. tb by facilitating uptake of divalent metals, such as iron and zinc (Ilghari et al., 2011). Regulatory binding sites for three divalent metal regulators, including; manganese transcriptional regulator (MntR), zinc uptake regulator (Zur), and iron-dependent regulator (IdeR) can be found in the ESX-3 core operon promoter (Ilghari et al., 2011).

\subsubsection{Manganese homeostasis}

Manganese $(\mathrm{Mn})$ is a metal element present in ample amount in nature (Farina et al., 2013). $\mathrm{Mn}$ is a cofactor of enzymes having diverse functionality from photosynthesis to detoxification. For example, $\mathrm{Mn}$ is also involved in the synthesis and degradation of hydrogen peroxide $\left(\mathrm{H}_{2} \mathrm{O}_{2}\right)$ superoxide, and free radicals (Juttukonda and Skaar, 2015). The oxidative burst is an effective bactericidal strategy against intracellular pathogens (Kumar et al., 2011). It has been previously reported that the manganese transcription regulator (MntR) regulates Mn homoeostasis (Zondervan et al., 2018). Mn is necessary for $M$. tb growth in human and/or mouse macrophages due to its restricted availability in the phagosome (Zondervan et al., 2018). Mn transport is required for virulence and the breakdown of oxygen radicals (Zondervan et al., 2018).

MntR regulates WhiB6, which regulates espACD and some DevR (previously known as DosR)-regulated genes (Zondervan et al., 2018). DevR is the main regulator of dormancy, while espACD is involved in pore formation. Zondervan et al. proposed that in $M$. marinum holo-WhiB6 positively regulates the ESX-1 operon upon reaction with ROS and NO (Zondervan et al., 2018) and Apo-WhiB6 and WhiB6-DNIC are formed. Both apo-WhiB6 and WhiB6-DNIC activate DevRregulated genes to shift metabolism to the dormancy phase by maintaining energy and redox homeostasis (Zondervan et al., 2018). MntR also acts as an anti-toxin in the toxin-antitoxin system of RelJ and RelK. The VapBC26 and VapB30 toxin-antitoxin system also requires $\mathrm{Mg}$ or $\mathrm{Mn}$ for their ribonuclease activity to inhibit growth (Zondervan et al., 2018). These results indicate the significance of $\mathrm{Mn}$ in regulation of $M$. tb growth.

\subsubsection{Zinc Homeostasis}

Zinc $(Z n)$ is used as a cofactor for many enzymes and DNA binding proteins (Zondervan et al., 2018). The most common enzymes of $M$. th that use $\mathrm{Zn}$ as cofactor, include: alcohol dehydrogenase (Stélandre et al., 1992), carbonic anhydrase (Covarrubias et al., 2005) and the Cu-Zn dependent superoxide dismutase (SodC) (Wu et al., 1998). Zn-dependent proteins in M. tb are involved in the biosynthesis of leucine (Koon et al., 2004), inositol-1-phosphate (Norman et al., 2002), glycosylphosphatidylinositol (Urbaniak et al., 2005) and degradation of mycothiol-S-conjugates (Steffek et al., 2003). These Zn-dependent proteins are also involved in DNA replication (Bhaduri et al., 1998) and removal of N-terminal methionine residues from some proteins post translation (Addlagatta et al., 2005). 
$\mathrm{Zn}$ is the only redox-stable divalent metal (Zondervan et al., 2018). This all shows that $\mathrm{Zn}$ is an important factor in pathogenicity of $M$. $t b$. Therefore, this bacterium must contain efficient zinc uptake systems. However, similar to other transition metals, zinc is also toxic at higher concentrations (Nies, 2007). There is a need for transport systems that remove superfluous cytoplasmic $\mathrm{Zn}^{2+}$ by efflux. Uncontrolled uptake and efflux reactions might lead to energy-wasting futile cycles and, thus, tight regulation is required to sense cytoplasmic (Bhaduri et al., 1998) $\mathrm{Zn}^{2+}$ concentration and use this information to control both expression of genes for and flux of transport systems (Nies, 2007). Zn homeostasis is controlled by Zur (FurB), a $\mathrm{Zn}^{2+}$-dependent repressor. Zur directly regulates 8 transcriptional units of $M$. tb. Studies have shown that 32 genes were upregulated in Zur knockout strains of which 24 belong to eight transcriptional units. Zur expression is controlled by $\mathrm{SmtB}$, which is a transcriptional repressor. SmtB is located upstream of Zur in its co-operon. SmtB expression was inactivated upon binding to $\mathrm{Zn}^{2+}$, so that Zur could perform its function (Zondervan et al., 2018).

\subsubsection{Control of iron homeostasis in macrophages}

Iron is a necessary micro-element for the survival and normal growth of $M$. tb (Sritharan, 2016). Iron is a part of the pathogen's electron transport chain (Sritharan, 2016) and a co-factor of several enzymes involved in respiration and DNA replication (Pandey and Rodriguez, 2014). To limit the iron acquisition by $M$. $t b$, the host cell produces natural resistance-associated macrophage protein 1 (Nramp1), which inhibits the transport of iron to the phagosome to restrict pathogen survival (Johnson and Wessling-Resnick, 2012). However, M. to can take up iron without expending energy by diffusion via porins that channel ferricarboxymycobactin through the envelope to membrane or cytoplasmic-associated ferrireductase (Banerjee et al., 2011). It is proposed that mycobactins may diffuse out through lipid membranes and travel as lipid vesicles in the host cell and sequester iron. These structures may recycle back to interact with the Mycobacterium. Carboxymycobactins are excreted, possibly by the Esx-3 of the type VII secretory system (T7SS). Esx-3 is one of the five clusters of T7SS which is involved in the homeostatic regulation $\mathrm{Fe}$ and $\mathrm{Zn}$ in $M$. tb (Tufariello et al., 2016). Externally, the carboxymycobactins bind with available iron from transferrin. Porins and $A B C$ transporters may allow the import of these ironloaded carboxymycobactins (Banerjee et al., 2011). The host cell addresses this situation by producing siderocalins, such as lipocalin-2, that can bind to and inactivate mycobactin, interfering with mycobacterial iron acquisition.

Iron is stored in bacterioferritin and a ferritin-like protein inside the mycobacterial cell (Reddy et al., 2012). These proteins are required for replication in macrophages to store iron and to limit excess iron, as excess stored iron causes oxidative damage by the Fenton reaction (Reddy et al., 2012). M. tb ironresponsive gene expression is balanced by the iron-dependent regulator, IdeR (Reddy et al., 2012). This protein can act both as an activator and a repressor, depending on type of mycobacterial promoter to bind with. It also acts as a repressor within promoters of mycobactin synthesis genes. It also inhibits the expression of these genes due to high iron concentration. In the promoter regions of iron storage genes, it acts as an activator by stimulating the expression of 
these genes at high iron concentrations, thus avoiding iron-stimulated oxidative damage (Reddy et al., 2012).

\subsubsection{Redox-potentiated proteins}

Another group of iron regulator proteins, WhiB, leads to iron homeostasis. WhiB3 regulates virulence-associated lipids, such as sulfolipids, diacyltrehaloses, and polyacyltrehaloses, all of which stimulate both higher pro- and anti-inflammatory cytokine levels and function in maintaining reduction-oxidation (redox) (Zheng et al., 2012). These proteins aid in the survival of $M$. $t b$ by retaining redox potential in the face of ROS and NOS. WhiB4 repression is associated with the oxidative stress response by activating antioxidant systems, including alkyl hydroperoxidase (ahpC-ahpD) and rubredoxins (rubA-rubB) (Chawla et al., 2012).

\subsection{Survival in the granuloma}

The granuloma provides a safe haven for $M$. tb to survive. Recent studies have shown that $M$. tb-infected macrophages shift from M1 to M2 (Sica et al., 2015). This shift helps $M$. tb to survive, as M2 macrophages provide a more conducive environment for intracellular pathogen(s) (Sica et al., 2015). M2 macrophages are also converted to foamy macrophages of the granuloma, which can host $M$. tb for longer periods. These macrophages are also believed to be involved in angiogenesis by releasing growth factors, such as vascular endothelial growth factor (VEGF). This angiogenesis allows oxygenation of some parts of the granuloma to promote survival and reactivation of TB (McClean and Tobin, 2016). The shift from M1 to M2 is mostly induced by the cytokines IL-4, IL-13, IL-10, and TGF- $\beta$ (Sica et al., 2015). IL-10 produces an anti-inflammatory response that inhibits the formation of dense and hypoxic, fibrotic granulomas (Cyktor et al., 2013; Silva Miranda et al., 2012). When $M$. tb encounters low oxygen tension in the granuloma, the DosS/DosT-DosR genes of the two-component systems and hspX (acr, Rv2031c) are activated, leading the pathogen into dormancy phase (Kumar et al., 2007). Further, the hspX chaperonin activity assists in refolding proteins that are damaged by low oxygen tension. These refolded proteins demonstrate a reactive response in the latently-infected TB patient (Taylor et al., 2012).

\subsection{Dormancy adaptation: Two-component systems (TCS)}

Dormancy is a highly-refined immune evasion strategy adopted by $M$. tb to persist for indefinite period of time (Peddireddy et al., 2017). To better understand the host-pathogen interaction during dormancy, researchers have identified various factors by which $M$. th tolerates the environmental changes elicited by the host's immune system (Chaves et al., 2015).

The two-component systems (TCS) of $M$. tb sense and adapt to different environmental conditions (Sharma and Tyagi, 2016). The well-recognized TCS in M. $t b$ is DevR-DevS or DosR-DosS, which is activated by hypoxia-inducing gasses (e.g. nitric oxide or carbon monoxide (Bretl et al., 2011)) and vitamin C (Taneja et al., 2010). DevR is also a main regulator for $M$. tb adaptation in the dormant state during infection (Sharma and Tyagi, 2016). Mehra et al. (2015) used a macaque model of tuberculosis to show that long-term persistence of 
DevR knockout strain infection was compromised. Moreover, $M$. tb dormancy can be correlated to inhibition of respiration, as the regulon is induced by hypoxia, $\mathrm{NO}$, and CO, which are the inhibitors of aerobic respiration (Drancourt, 2009). The study of Leistikow et al. (Leistikow et al., 2010) showed that the DosR regulon helps $M$. tb to survive during anaerobic dormancy by maintaining ATP levels and balancing the redox state (NAD/NADH ratio) of the cell.

\subsection{Toxin anti-toxin system}

The toxin anti-toxin (TAT) system is another success story of $M$. tb survival. Toxins are stable and usually target some important cellular function. On the other hand, anti-toxins are unstable but bind and inhibit toxins (Ramage et al., 2009). TAT genes mostly have a two-gene operon. Therefore, the anti-toxin may act as an operon repressor whose degradation is required to liberate the production and function of the toxin (Ramage et al., 2009). Until or unless environmental influences break the complex to release toxins within bacterial cell, these toxins cannot interact with host cellular component(s). The toxins destroy the mRNA of the pathogens' own growth-promoting genes, making the bacterial cell dormant. The system is functionally analogous to the host miRNA-mRNA interaction. Finally, dormancy is helpful for the persistence of $M$. tb within host, as dormant bacteria are more resistant to stress than active bacteria (Ramage et al., 2009).

\section{Micro RNA-mediated immune response modulation}

MicroRNA (miRNA) are studied and utilized as medicine, especially for diseases in which the pathogen is intracellular and seizes the host machinery (Liang et al., 2017). It has been hypothesized that microRNAs (miRNAs) could be the novel target(s) of interaction to regulate host gene expression (Wang et al., 2014), as shown in Figure 2. M. tb mainly manipulates miRNAs that are directly involved in altering innate and adaptive immune functions. Zhou et al., (Zhou et al., 2018) have also declared that miRNA and small non-coding RNA have a crucial role in the pathogenesis of $M$. tb infection. These RNAs helps bacterial cells in the induction of infection, cell structural remodeling and boosting of innate/adaptive immunity. Recent work has also shown that some host miRNA, such as miR146a, increases after infection. This miRNA is involved in intra-macrophage mycobacterial survival by targeting TRAF6 mRNA and, therefore, suppressing iNOS expression ( $\mathrm{Li}$ et al., 2016). Contrary to miR146a, the level of miR144, which is involved in targeting tumor progression locus 2 (TpL2, also known as MAP3K8) and Extracellular Signal Regulated Kinase (ERK) signaling, decreases during M. tb infection (Liu, 2016). Both MAP3K8 and ERK signaling are important for macrophages to clear $M$. tb infection, through the enhanced production of TNF- $\alpha$, IL1- $\beta$, and IL-6 (Liu, 2016). These studies suggest that miRNAs could be used as biomarkers of active tuberculosis. Ahmed et al., (Ahmed et al., 2016; Ahmed et al., 2018) has revealed the role of small RNA (sRNAs) in Brucella pathogenesis. These sRNAs interact with host cell gene expression transcriptional product i.e. mRNA and in this way modulate the posttranscriptional modifications.

Gene transcription in a normal eukaryotic cell is dependent on several epigenetic factors. These factors lead to chromatin conformational modification(s). 
Therefore, the cellular physiological response is the collective output of chromatin organization and the related epigenetic change(s) of an individual gene (epigenome) (Khosla et al., 2016). Moreover, during cellular growth and differentiation, the cell's microenvironment has a tremendous influence on the cell epigenome (Khosla et al., 2016). Pathogenic M. tb has evolved strategies to manipulate the host epigenome for its survival by hijacking the transcriptional machinery of the host (Khosla et al., 2016). M. tb uses Enhanced intracellular survival (Eis) protein to negatively influence normal host cellular mechanisms, including JNK-dependent autophagy, phagosome maturation, and ROS generation, through its acetylating activity (Kim et al., 2012; Liang et al., 2017). Other host genome modulation proteins, such as Rv1988 and Rv2966c, have been reported to be histone methyltransferases (Khosla et al., 2016). Rv1988 targets host defensive proteins, such as reactive oxygen species producing proteins, NOX1, NOX4, and NOS2, and TNF receptor-associated factor 3 (TRAF3) (Khosla et al., 2016). It has been suggested that Rv1988 and Rv2966c are a first line of attack during $M$. tb infection by impairing the ability to mount an immune response, then subverting host signaling cascades and paralyzing phagosomal machinery. This ultimately helps tilt the balance in favor of the pathogen within the hostile environment of the host (Khosla et al., 2016).

\section{Conclusion}

This review summarizes various intracellular immune escape mechanisms of $M$. tb that manipulates host protective immunity by influencing physiological mechanisms and signaling pathways. $M$. tb has been considered a global havoc because of the lack of effective therapeutic strategies to eradicate it. Big leaps in the understanding of TB immunology and cell biology become necessary to identify new remedial avenues. However, we must not underestimate the power of natural selection for the survival of $M$. tb.

\section{Compliance with ethics guidelines:}

Muhammad Ahsan Naeem, Waqas Ahmad, Rohit Tyagi, Qaiser Akram, Muhammad Younus and Xilin Liu declare that they have no conflict of interest or financial conflicts to disclose.

\section{References}

Addlagatta, A., Quillin, M.L., Omotoso, O., Liu, J.O., and Matthews, B.W. (2005). Identification of an SH3-binding motif in a new class of methionine aminopeptidases from Mycobacterium tuberculosis suggests a mode of interaction with the ribosome. Biochemistry 44, 7166-7174.

Ahmed, W., Zheng, K., Liu, Z. F. (2016). Establishment of chronic infection: Brucella's stealth strategy. Frontiers in cellular and infection microbiology, 6, 30.

Ahmed, W., Hafeez, M. A., Mahmood, S. (2018). Identification and functional characterization of bacterial small non-coding RNAs and their target: A review. Gene Reports, 10, 167-176.

Augenstreich, J., Arbues, A., Simeone, R., Haanappel, E., Wegener, A., Sayes, F., Le Chevalier, F., Chalut, C., Malaga, W., and Guilhot, C. (2017). ESX-1 and phthiocerol dimycocerosates of Mycobacterium tuberculosis act in concert to 
cause phagosomal rupture and host cell apoptosis. Cellular microbiology 19, e12726.

Banerjee, S., Farhana, A., Ehtesham, N.Z., and Hasnain, S.E. (2011). Iron acquisition, assimilation and regulation in mycobacteria. Infection, Genetics and Evolution 11, 825-838.

Bhaduri, T., Bagui, T.K., Sikder, D., and Nagaraja, V. (1998). DNA topoisomerase I from Mycobacterium smegmatis An enzyme with distinct features. Journal of Biological Chemistry 273, 13925-13932.

Bowdish, D.M., Sakamoto, K., Kim, M.-J., Kroos, M., Mukhopadhyay, S., Leifer, C.A., Tryggvason, K., Gordon, S., and Russell, D.G. (2009). MARCO, TLR2, and CD14 are required for macrophage cytokine responses to mycobacterial trehalose dimycolate and Mycobacterium tuberculosis. PLoS Pathog 5, e1000474.

Bretl, D.J., Demetriadou, C., and Zahrt, T.C. (2011). Adaptation to environmental stimuli within the host: two-component signal transduction systems of Mycobacterium tuberculosis. Microbiology and Molecular Biology Reviews 75, 566-582.

Cambier, C., Falkow, S., and Ramakrishnan, L. (2014). Host evasion and exploitation schemes of Mycobacterium tuberculosis. Cell 159, 1497-1509.

Chaves, A.S., Rodrigues, M.F., Mattos, A.M.M., and Teixeira, H.C. (2015). Challenging Mycobacterium tuberculosi $s$ dormancy mechanisms and their immunodiagnostic potential. Brazilian Journal of Infectious Diseases 19, 636-642.

Chawla, M., Parikh, P., Saxena, A., Munshi, M., Mehta, M., Mai, D., Srivastava, A.K., Narasimhulu, K., Redding, K.E., and Vashi, N. (2012). Mycobacterium tuberculosis WhiB4 regulates oxidative stress response to modulate survival and dissemination in vivo. Molecular microbiology 85, 1148-1165.

Chen, X., Sakamoto, K., Quinn, F.D., Chen, H., and Fu, Z. (2015). Lack of intracellular replication of $M$. tuberculosis and $M$. bovis BCG caused by delivering bacilli to lysosomes in murine brain microvascular endothelial cells. Oncotarget 6, 32456.

Colangeli, R., Haq, A., Arcus, V.L., Summers, E., Magliozzo, R., McBride, A., Mitra, A., Radjainia, M., Khajo, A., and Jacobs, W. (2009). The multifunctional histone-like protein Lsr2 protects mycobacteria against reactive oxygen intermediates. Proceedings of the National Academy of Sciences 106, 4414-4418.

Comas, I., Coscolla, M., Luo, T., Borrell, S., Holt, K.E., Kato-Maeda, M., Parkhill, J., Malla, B., Berg, S., and Thwaites, G. (2013). Out-of-Africa migration and Neolithic coexpansion of Mycobacterium tuberculosis with modern humans. Nature genetics 45, 1176-1182.

Covarrubias, A.S., Larsson, A.M., Högbom, M., Lindberg, J., Bergfors, T., Björkelid, C., Mowbray, S.L., Unge, T., and Jones, T.A. (2005). Structure and function of carbonic anhydrases from Mycobacterium tuberculosis. Journal of Biological Chemistry 280, 18782-18789.

Cyktor, J.C., Carruthers, B., Kominsky, R.A., Beamer, G.L., Stromberg, P., and Turner, J. (2013). IL-10 inhibits mature fibrotic granuloma formation during Mycobacterium tuberculosis infection. The Journal of Immunology 190, 2778-2790. 
De Libero, G., and Mori, L. (2014). The T-cell response to lipid antigens of Mycobacterium tuberculosis. Frontiers in immunology 5, 219.

Dean, A.S., Forcella, S., Olea-Popelka, F., Idrissi, A.E., Glaziou, P., Benyahia, A., Mumford, E., Erlacher-Vindel, E., Gifford, G., and Lubroth, J. (2018). A roadmap for zoonotic tuberculosis: a One Health approach to ending tuberculosis. The Lancet Infectious diseases 18, 137.

Deretic, V., Singh, S., Master, S., Harris, J., Roberts, E., Kyei, G., Davis, A., De Haro, S., Naylor, J., and Lee, H.H. (2006). Mycobacterium tuberculosis inhibition of phagolysosome biogenesis and autophagy as a host defence mechanism. Cellular microbiology 8, 719-727.

Drancourt, M. (2009). Mycobacterium: genomics and molecular biology. The Lancet Infectious Diseases 9, 535.

Ehrt, S., and Schnappinger, D. (2009). Mycobacterial survival strategies in the phagosome: defence against host stresses. Cellular microbiology 11, 1170-1178.

Ernst, J.D. (1998). Macrophage receptors for Mycobacterium tuberculosis. Infection and immunity 66, 1277-1281.

Farina, M., Avila, D.S., Da Rocha, J.B.T., and Aschner, M. (2013). Metals, oxidative stress and neurodegeneration: a focus on iron, manganese and mercury. Neurochemistry international 62, 575-594.

Ferguson, J. S., Weis, J. J., Martin, J. L., \& Schlesinger, L. S. (2004). Complement protein C3 binding to Mycobacterium tuberculosis is initiated by the classical pathway in human bronchoalveolar lavage fluid. Infection and Immunity, 72(5), 2564-2573.

Ferrari, G., Langen, H., Naito, M., and Pieters, J. (1999). A coat protein on phagosomes involved in the intracellular survival of mycobacteria. Cell 97, 435-447.

Ferraris, D.M., Miggiano, R., Rossi, F., and Rizzi, M. (2018). Mycobacterium tuberculosis molecular determinants of infection, survival strategies, and vulnerable targets. Pathogens 7, 17.

Gidon, A., Åsberg, S.E., Louet, C., Ryan, L., Haug, M., and Flo, T.H. (2017). Correction: Persistent mycobacteria evade an antibacterial program mediated by phagolysosomal TLR7/8/MyD88 in human primary macrophages. PLoS pathogens 13, e1006712.

Harding, C.V., and Boom, W.H. (2010). Regulation of antigen presentation by Mycobacterium tuberculosis: a role for Toll-like receptors. Nature Reviews Microbiology 8, 296-307.

Hossain, M., and Norazmi, M.-N. (2013). Pattern recognition receptors and cytokines in Mycobacterium tuberculosis infection-the double-edged sword? BioMed research international 2013.

Hurley, D., McCusker, M. P., Fanning, S., Martins, M. (2014). Salmonella-host interactions-modulation of the host innate immune system. Frontiers in immunology, 5, 481.

Ilghari, D., Lightbody, K.L., Veverka, V., Waters, L.C., Muskett, F.W., Renshaw, P.S., and Carr, M.D. (2011). Solution Structure of the Mycobacterium tuberculosis EsxG. EsxH Complex Functional Implications And Comparisons With Other M. Tuberculosis Esx Family Complexes. Journal of Biological Chemistry 286, 29993-30002. 
Jayachandran, R., Sundaramurthy, V., Combaluzier, B., Mueller, P., Korf, H., Huygen, K., Miyazaki, T., Albrecht, I., Massner, J., and Pieters, J. (2007). Survival of mycobacteria in macrophages is mediated by coronin 1-dependent activation of calcineurin. Cell 130, 37-50.

Johnson, E.E., and Wessling-Resnick, M. (2012). Iron metabolism and the innate immune response to infection. Microbes and infection 14, 207-216.

Juttukonda, L.J., and Skaar, E.P. (2015). Manganese homeostasis and utilization in pathogenic bacteria. Molecular microbiology 97, 216-228.

Khosla, S., Sharma, G., and Yaseen, I. (2016). Learning epigenetic regulation from mycobacteria. Microbial Cell 3, 92.

Kim, K.H., An, D.R., Song, J., Yoon, J.Y., Kim, H.S., Yoon, H.J., Im, H.N., Kim, J., Lee, S.J., and Kim, K.-H. (2012). Mycobacterium tuberculosis Eis protein initiates suppression of host immune responses by acetylation of DUSP16/ MKP-7. Proceedings of the National Academy of Sciences 109, 7729-7734.

Koon, N., Squire, C.J., and Baker, E.N. (2004). Crystal structure of LeuA from Mycobacterium tuberculosis, a key enzyme in leucine biosynthesis. Proceedings of the National Academy of Sciences 101, 8295-8300.

Kumar, A., Farhana, A., Guidry, L., Saini, V., Hondalus, M., and Steyn, A.J. (2011). Redox homeostasis in mycobacteria: the key to tuberculosis control? Expert reviews in molecular medicine 13.

Kumar, A., Toledo, J.C., Patel, R.P., Lancaster, J.R., and Steyn, A.J. (2007). Mycobacterium tuberculosis DosS is a redox sensor and DosT is a hypoxia sensor. Proceedings of the National Academy of Sciences 104, 11568-11573.

Lang, R. (2013). Recognition of the mycobacterial cord factor by Mincle: relevance for granuloma formation and resistance to tuberculosis. Frontiers in immunology 4, 5 .

Leistikow, R.L., Morton, R.A., Bartek, I.L., Frimpong, I., Wagner, K., and Voskuil, M.I. (2010). The Mycobacterium tuberculosis DosR regulon assists in metabolic homeostasis and enables rapid recovery from nonrespiring dormancy. Journal of bacteriology 192, 1662-1670.

Levitte, S., Adams, K.N., Berg, R.D., Cosma, C.L., Urdahl, K.B., and Ramakrishnan, L. (2016). Mycobacterial acid tolerance enables phagolysosomal survival and establishment of tuberculous infection in vivo. Cell host \& microbe 20, 250-258.

Li, F., Feng, L., Jin, C., Wu, X., Fan, L., Xiong, S., and Dong, Y. (2018). LpqT improves mycobacteria survival in macrophages by inhibiting TLR2 mediated inflammatory cytokine expression and cell apoptosis. Tuberculosis 111, 57-66.

Li, M., Wang, J., Fang, Y., Gong, S., Li, M., Wu, M., Lai, X., Zeng, G., Wang, Y., and Yang, K. (2016). Corrigendum: microRNA-146a promotes mycobacterial survival in macrophages through suppressing nitric oxide production. Scientific Reports 6.

Liang, M., Habib, Z., Sakamoto, K., Chen, X., and Cao, G. (2017). Mycobacteria and autophagy: many questions and few answers. Curr Issues Mol Biol 21, 63-72.

Lisdawati, V., Puspandari, N., Rif'ati, L., Soekarno, T., Melatiwati, M., Syamsidar, K., Ratnasari, L., Izzatun, N., and Parwati, I. (2015). Molecular epidemiology study of Mycobacterium tuberculosis and its susceptibility to anti-tuberculosis drugs in Indonesia. BMC infectious diseases 15, 366. 
Liu, H. (2016). Down-regulation of miR-144 after Mycobacterium tuberculosis infection promotes inflammatory factor secretion from macrophages through the Tpl2/ERK pathway. Cellular and Molecular Biology 62, 87-93.

Master, S.S., Rampini, S.K., Davis, A.S., Keller, C., Ehlers, S., Springer, B., Timmins, G.S., Sander, P., and Deretic, V. (2008). Mycobacterium tuberculosis prevents inflammasome activation. Cell host \& microbe 3, 224-232.

McClean, C.M., and Tobin, D.M. (2016). Macrophage form, function, and phenotype in mycobacterial infection: lessons from tuberculosis and other diseases. Pathogens and disease 74.

Meena, L.S. (2010). Survival mechanisms of pathogenic Mycobacterium tuberculosis H37Rv. The FEBS journal 277, 2416-2427.

Mehra, S., Foreman, T.W., Didier, P.J., Ahsan, M.H., Hudock, T.A., Kissee, R., Golden, N.A., Gautam, U.S., Johnson, A.-M., and Alvarez, X. (2015). The DosR regulon modulates adaptive immunity and is essential for Mycobacterium tuberculosis persistence. American journal of respiratory and critical care medicine 191, 1185-1196.

Mortaz, E., Adcock, I.M., Tabarsi, P., Masjedi, M.R., Mansouri, D., Velayati, A.A., Casanova, J.-L., and Barnes, P.J. (2015). Interaction of pattern recognition receptors with Mycobacterium tuberculosis. Journal of clinical immunology 35, 1-10.

Naffin-Olivos, J.L., Georgieva, M., Goldfarb, N., Madan-Lala, R., Dong, L., Bizzell, E., Valinetz, E., Brandt, G.S., Yu, S., and Shabashvili, D.E. (2014). Mycobacterium tuberculosis Hip1 modulates macrophage responses through proteolysis of GroEL2. PLoS Pathog 10, e1004132.

Nies, D.H. (2007). Bacterial transition metal homeostasis. In Molecular microbiology of heavy metals (Springer), pp. 117-142.

Norman, R.A., McAlister, M.S., Murray-Rust, J., Movahedzadeh, F., Stoker, N.G., and McDonald, N.Q. (2002). Crystal structure of inositol 1-phosphate synthase from Mycobacterium tuberculosis, a key enzyme in phosphatidylinositol synthesis. Structure 10, 393-402.

Pandey, R., and Rodriguez, G.M. (2014). IdeR is required for iron homeostasis and virulence in $\mathrm{M}$ ycobacterium tuberculosis. Molecular microbiology 91, 98-109.

Patin, E.C., Geffken, A.C., Willcocks, S., Leschczyk, C., Haas, A., Nimmerjahn, F., Lang, R., Ward, T.H., and Schaible, U.E. (2017). Trehalose dimycolate interferes with FcyR-mediated phagosome maturation through Mincle, SHP-1 and FcyRIIB signalling. PloS one 12, e0174973.

Peddireddy, V., Doddam, S.N., and Ahmed, N. (2017). Mycobacterial dormancy systems and host responses in tuberculosis. Frontiers in immunology 8, 84 .

Phillips, B.L., Gautam, U.S., Bucsan, A.N., Foreman, T.W., Golden, N.A., Niu, T., Kaushal, D., and Mehra, S. (2017). LAG-3 potentiates the survival of Mycobacterium tuberculosis in host phagocytes by modulating mitochondrial signaling in an in-vitro granuloma model. PloS one 12, e0180413.

Pieters, J. (2008). Mycobacterium tuberculosis and the macrophage: maintaining a balance. Cell host \& microbe 3, 399-407.

Purdy, G.E., Pacheco, S., Turk, J., and Hsu, F.-F. (2013). Characterization of mycobacterial triacylglycerols and monomeromycolyl diacylglycerols from Mycobacterium smegmatis biofilm by electrospray ionization multiple-stage and 
high-resolution mass spectrometry. Analytical and bioanalytical chemistry 405 , 7415-7426.

Ramage, H.R., Connolly, L.E., and Cox, J.S. (2009). Comprehensive functional analysis of Mycobacterium tuberculosis toxin-antitoxin systems: implications for pathogenesis, stress responses, and evolution. PLoS Genet 5, e1000767.

Reddy, P.V., Puri, R.V., Khera, A., and Tyagi, A.K. (2012). Iron storage proteins are essential for the survival and pathogenesis of Mycobacterium tuberculosis in THP-1 macrophages and the guinea pig model of infection. Journal of bacteriology 194, 567-575.

Rohde, K., Yates, R.M., Purdy, G.E., and Russell, D.G. (2007). Mycobacterium tuberculosis and the environment within the phagosome. Immunological reviews 219, 37-54.

Saurabh, A., Chakraborty, S., Kumar, P., Mohan, A., Bhatnagar, A.K., Rishi, N., and Mitra, D.K. (2018). Inhibiting HLA-G restores IFN- $\gamma$ and TNF- $\alpha$ producing T cell in pleural tuberculosis. Tuberculosis 109, 69-79.

Saviola, B. (2010). All stressed out: mycobacterial responses to stress. Current Research, Technology, and Education Topics in Applied Microbiology and Microbial Biotechnology Microbiology Book Series Edition, ed Mendez-Vilas A, editor(Formatex Research Center, 545-549.

Saviola, B. (2013). Mycobacterium tuberculosis adaptation to survival in a human host (ISBN).

Sharma, S., and Tyagi, J.S. (2016). Mycobacterium tuberculosis DevR/DosR dormancy regulator activation mechanism: dispensability of phosphorylation, cooperativity and essentiality of $\alpha 10$ helix. PloS one 11, e0160723.

Sica, A., Erreni, M., Allavena, P., and Porta, C. (2015). Macrophage polarization in pathology. Cellular and molecular life sciences 72, 4111-4126.

Silva Miranda, M., Breiman, A., Allain, S., Deknuydt, F., and Altare, F. (2012). The tuberculous granuloma: an unsuccessful host defence mechanism providing a safety shelter for the bacteria? Clinical and Developmental Immunology 2012.

Singh, A., Gupta, R., Vishwakarma, R., Narayanan, P., Paramasivan, C., Ramanathan, V., and Tyagi, A.K. (2005). Requirement of the mymA operon for appropriate cell wall ultrastructure and persistence of Mycobacterium tuberculosis in the spleens of guinea pigs. Journal of bacteriology 187 , 4173-4186.

Sritharan, M. (2016). Iron homeostasis in Mycobacterium tuberculosis: mechanistic insights into siderophore-mediated iron uptake. Journal of bacteriology 198, 2399-2409.

Srivastava, S., and Ernst, J.D. (2014). Cell-to-cell transfer of M. tuberculosis antigens optimizes CD4 T cell priming. Cell host \& microbe 15, 741-752.

Steffek, M., Newton, G.L., Av-Gay, Y., and Fahey, R.C. (2003). Characterization of Mycobacterium tuberculosis mycothiol S-conjugate amidase. Biochemistry 42, 12067-12076.

Stélandre, M., Bosseloir, Y., De Bruyn, J., Maes, P., and Content, J. (1992). Cloning and sequence analysis of the gene encoding an NADP-dependent alcohol dehydrogenase in Mycobacterium bovis BCG. Gene 121, 79-86.

Swanson, J.A. (2002). The extraordinary phagosome. Nature 418, 286-287.

Tailleux, L., Neyrolles, O., Honoré-Bouakline, S., Perret, E., Sanchez, F., Abastado, J.-P., Lagrange, P.H., Gluckman, J.C., Rosenzwajg, M., and 
Herrmann, J.-L. (2003). Constrained intracellular survival of Mycobacterium tuberculosis in human dendritic cells. The Journal of Immunology 170, 1939-1948.

Taneja, N.K., Dhingra, S., Mittal, A., Naresh, M., and Tyagi, J.S. (2010). Mycobacterium tuberculosis transcriptional adaptation, growth arrest and dormancy phenotype development is triggered by vitamin C. PloS one 5, e10860.

Taylor, J.L., Wieczorek, A., Keyser, A.R., Grover, A., Flinkstrom, R., Karls, R.K., Bielefeldt-Ohmann, H., Dobos, K.M., and Izzo, A.A. (2012). HspX-mediated protection against tuberculosis depends on its chaperoning of a mycobacterial molecule. Immunology and cell biology 90, 945-954.

Torres, M., Ramachandra, L., Rojas, R.E., Bobadilla, K., Thomas, J., Canaday, D.H., Harding, C.V., and Boom, W.H. (2006). Role of phagosomes and major histocompatibility complex class II (MHC-II) compartment in MHC-II antigen processing of Mycobacterium tuberculosis in human macrophages. Infection and immunity $74,1621-1630$.

Tufariello, J. M., Chapman, J. R., Kerantzas, C. A., Wong, K. W., Vilchèze, C., Jones, C. M., ... \& Niederweis, M. (2016). Separable roles for Mycobacterium tuberculosis ESX-3 effectors in iron acquisition and virulence. Proceedings of the National Academy of Sciences, 113(3), E348-E357.

Urbaniak, M.D., Crossman, A., Chang, T., Smith, T.K., van Aalten, D.M., and Ferguson, M.A. (2005). The N-acetyl-D-glucosaminylphosphatidylinositol De-Nacetylase of glycosylphosphatidylinositol biosynthesis is a zinc metalloenzyme. Journal of Biological Chemistry 280, 22831-22838.

Walburger, A., Koul, A., Ferrari, G., Nguyen, L., Prescianotto-Baschong, C., Huygen, K., Klebl, B., Thompson, C., Bacher, G., and Pieters, J. (2004). Protein kinase $G$ from pathogenic mycobacteria promotes survival within macrophages. Science 304, 1800-1804.

Wang, J., Teng, J.L., Zhao, D., Ge, P., Li, B., Woo, P.C., and Liu, C.H. (2016). The ubiquitin ligase TRIM27 functions as a host restriction factor antagonized by Mycobacterium tuberculosis PtpA during mycobacterial infection. Scientific reports 6,34827 .

Wang, J., Wu, M., Wen, J., Yang, K., Li, M., Zhan, X., Feng, L., Li, M., and Huang, X. (2014). MicroRNA-155 induction by Mycobacterium bovis BCG enhances ROS production through targeting SHIP1. Molecular Immunology 62, 29-36.

Wang, Y., Zhong, H., Xie, X., Chen, C.Y., Huang, D., Shen, L., Zhang, H., Chen, Z.W., and Zeng, G. (2015). Long noncoding RNA derived from CD244 signaling epigenetically controls CD8+ T-cell immune responses in tuberculosis infection. Proceedings of the National Academy of Sciences 112, E3883-E3892.

Watson, R.O., Bell, S.L., MacDuff, D.A., Kimmey, J.M., Diner, E.J., Olivas, J., Vance, R.E., Stallings, C.L., Virgin, H.W., and Cox, J.S. (2015). The cytosolic sensor cGAS detects Mycobacterium tuberculosis DNA to induce type I interferons and activate autophagy. Cell host \& microbe 17, 811-819.

Wong, D., Bach, H., Sun, J., Hmama, Z., and Av-Gay, Y. (2011). Mycobacterium tuberculosis protein tyrosine phosphatase (PtpA) excludes host vacuolar-H+ATPase to inhibit phagosome acidification. Proceedings of the National Academy of Sciences 108, 19371-19376. 
Wu, C.H.H., Tsai-Wu, J.J., Huang, Y.T., Lin, C.Y., Lioua, G.G., and Lee, F.J.S. (1998). Identification and subcellular localization of a novel $\mathrm{Cu}, \mathrm{Zn}$ superoxide dismutase of Mycobacterium tuberculosis.

Zheng, F., Long, Q., and Xie, J. (2012). The function and regulatory network of WhiB and WhiB-like protein from comparative genomics and systems biology perspectives. Cell biochemistry and biophysics 63, 103-108.

Zhou, X., Li, X., \& Wu, M. (2018). miRNAs reshape immunity and inflammatory responses in bacterial infection. Signal transduction and targeted therapy, 3(1), $1-13$.

Zondervan, N.A., Van, J.D., Schaap, P.J., Martins, V.D.S., and Suarezdiez, M. (2018). Regulation of Three Virulence Strategies of Mycobacterium tuberculosis: A Success Story. International Journal of Molecular Sciences 19. 
